\title{
Design and Implementation of DMA Transfer Through PCI Express Gen3 Interface Based on FPGA
}

\author{
Yufei Zhang ${ }^{1, a}$, Hengzhu Liu ${ }^{1, b}$, Xiaodong Yi ${ }^{1, c}$, Xuan Zhu ${ }^{1, d}$ \\ ${ }^{1}$ Department of Computer, National University of Defense Technology, ChangSha, 410073,China \\ a yueyizhuying@gmail.com, b hengzhu_liu@263.net, 'xdong_yi@163.com, dzx00348711@163.com
}

Keywords: FPGA, PCI Express 3.0, DMA Read, DMA Write.

\begin{abstract}
The future requirements for bandwidth and scalability of big data has gone beyond the performance of the second generation I/O interconnect. Peripheral component interconnect express (PCI Express) 3.0 is the latest standard as one of the third generation I/O interconnect for expansion cards. The bit rate of this fully serial interface has reached 8 giga transfers per second(Gbps), which could meet the demand of future computing and communication platforms. In this paper, we designed and implemented the directly memory access (DMA) transfer of PCI Express Gen3 interface based on the Xilinx Virtex-7 FPGA Gen3 Integrated Block for PCI Express core. The proposed design has debugged and verified on the Xilinx Virtex-7 FPGA VC709 Connectivity Kit and could carry an average rate of $3.04 \mathrm{~GB} / \mathrm{s}$, this design provides up to $300 \%$ improvement over the similar design based on PCI Express Gen2 interface.
\end{abstract}

\section{Introduction}

With the development of cloud computing, internet of things and social network technology, the amount of data is growing and accumulating at a dramatically high speed. For example, Google accesses 400PB data per month through large-scale cluster and Map Reduce software. The rapid development of Big Data calls for a high speed of data transferring. In order to satisfy the demands of transferring rate, PCI Express is constantly developing. PCI Express 3.0 carries a data transferring rate of $8 \mathrm{Gbps}$ per lane and upgrades the coding scheme to $128 \mathrm{~b} / 130 \mathrm{~b}[1]$. It grows $3 \mathrm{Gbps}$ on date transferring rate and reduces the bandwidth overhead from $20 \%$ to $1.54 \%$ compared with PCI Express 2.0[2]. Since 2013, Xilinx, Altera, PLDA, NVIDA and other companies have released chips which in compliant with PCI Express 3.0 Base Specification, PCI Express 3.0 has been widely used in many fields[3], but currently these applications are not open source[4].

In this paper, we designed a PCI Express Gen3 interface through which the data could be transferred between the DDR3 in the computer and the SODIMM on the Xilinx VC709 development board. To improve the data transferring rata, we further designed a DMA controller. Overall, the major contributions of this paper are:

- We analyzed the upgrades of the Xilinx Virtex-7 FPGA Gen3 Integrated Block for PCI Express, and further designed a new PCI Express Gen3 interface based on this core.

- We designed a DMA controller based on the PCI Express Gen3 interface. The test results showed that this interface could carry a rate of $2.89 \mathrm{~GB} / \mathrm{s}$ of DMA read and $3.18 \mathrm{~GB} / \mathrm{s}$ of DMA write.

The remainder of this paper is organized as follows: In Section 2, we summarize the improvement of the Xilinx FPGA PCI Express core. Section 3 describes the design and implementation of PCI Express Gen3 DMA, followed by evaluation in Section 4, related work in Section 5, and conclusions in Section 6. 


\section{The improvement of Xilinx PCI Express Gen3 IP core}

In this section, we made comparison between the Xilinx PCI Express Gen3 and Gen2 IP Cores. Through the analysis, we found that the performance advantages of theVirtex-7 PCI Express Gen3 Integrated Block originate from improvements in client interface, transaction layer packets(TLP) descriptor format and data alignment mode. Such a perspective provided us with a clear guidance of the design of the PCI Express Gen3 interface.

Client interface. TheVirtex-7 PCI Express Gen3 Integrated Block consists of four AXI4-Stream Interfaces o receive and transfer transactions while the Virtex-6 PCI Express Gen2 Integrated Block only have two AXI4-Stream Interfaces, thus the further one could simplify the transactions. Figure 1 shows the two different client-side interfaces.
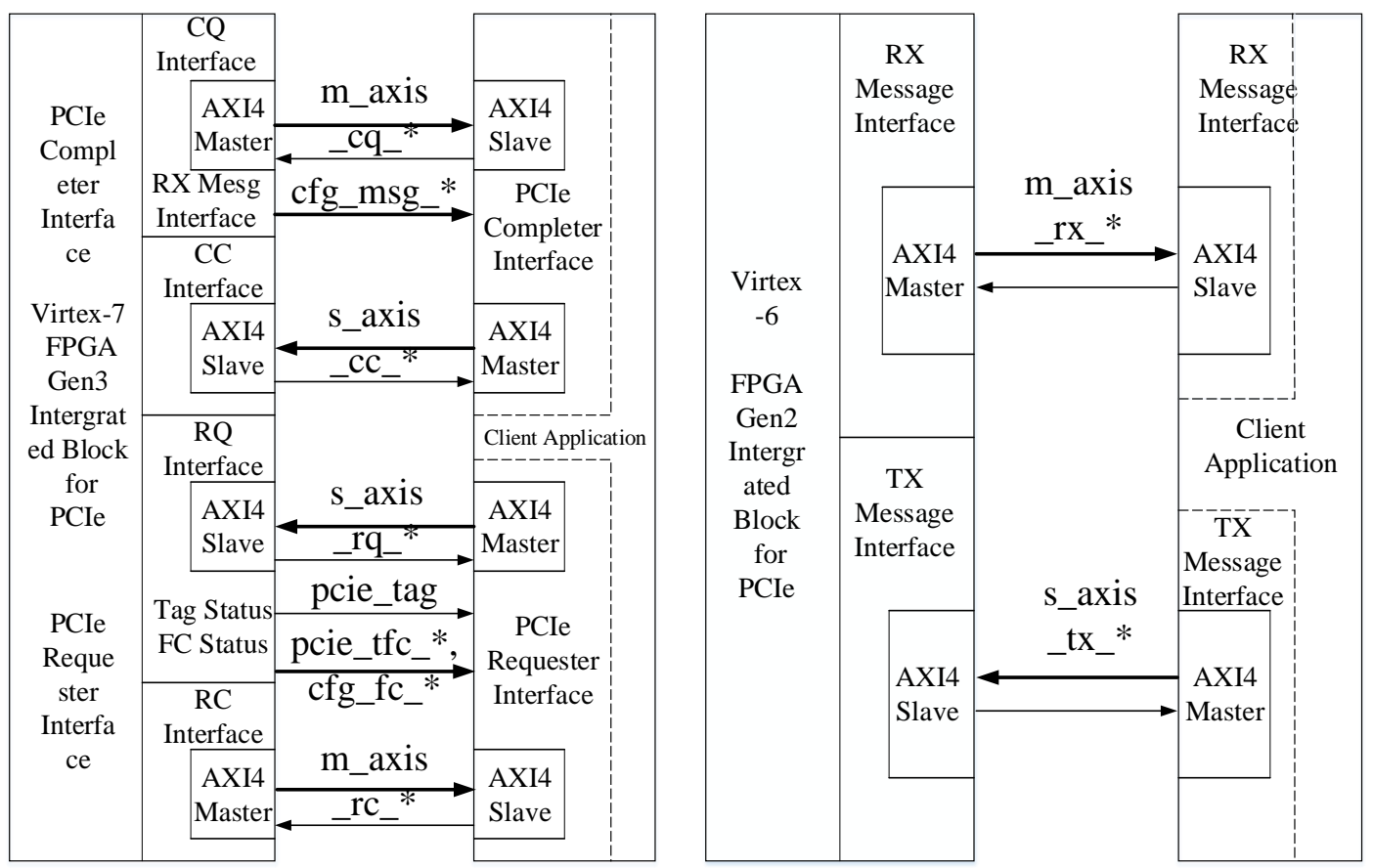

Fig 1: Block Diagram of Virtex-7 FPGA Gen3 Integrated Block Client Interfaces[5] and Virtex-6 FPGA Gen2 Integrated Block Client Interfaces[6]

- Completer reQuest (CQ) Interface: The interface delivers all of the request packets from the link to user application.

- Completer Completion (CC) Interface: The interface transmits the response packets generated by the user application. It can accept and send packets at the same time.

- Requester reQuest (RQ) Interface: The interface transmits the request packets generated by the user application.

- Requester Completion (RC) Interface: The interface delivers the response packets from the link.

The transactions on the Requester Interface and Completer Interface are similar, each of them has two separate interfaces, one for the different direction. Each of them is compliant with the AXI4-Stream protocol, and the width can be configured as 256,128, or 64 bits. The only difference is that the roles of the user application and the core are reversed.

TLP descriptor format. The Virtex-7 PCI Express Gen3 Integrated Block transmits and receives data as an independent packet. Every packet begin with a descriptor and can be followed with payload. The descriptor is sent in the first 4 double word (DW) and always 4DW long. During the first beat of the 256-bit interface, the descriptor is transferred. The format of memory request types descriptor is illustrated in Figure 2. The format of Figure 3 applies in the Virtex-6 PCI Express Gen3 Integrated Block interfaces. 


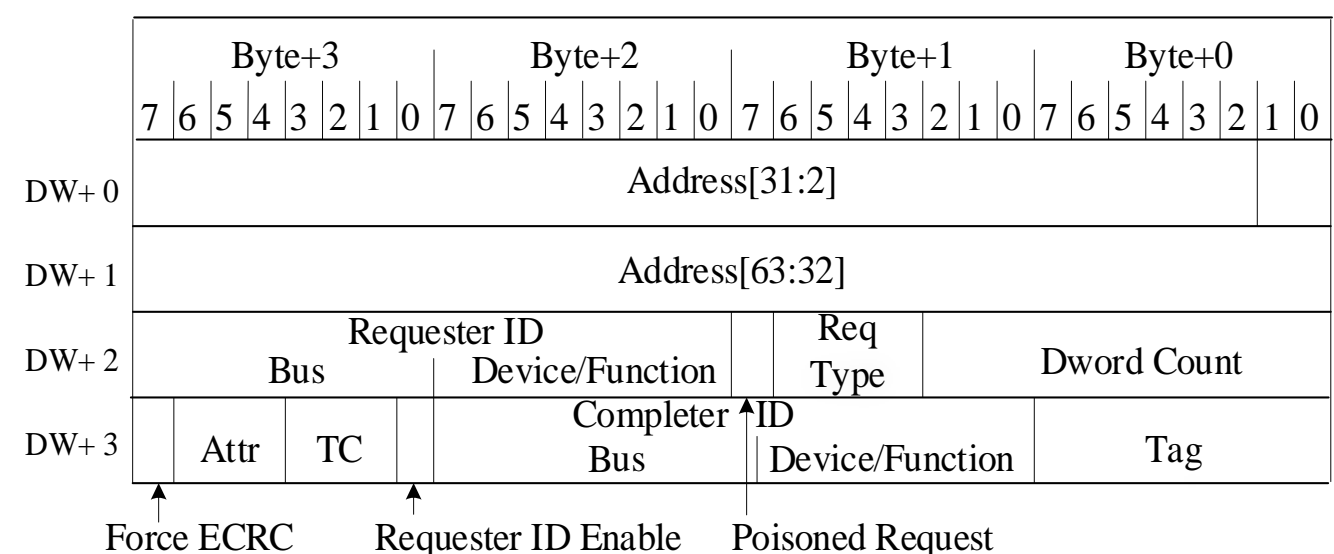

Fig 2: PCI Express Base Specification 3.0 Byte Order[1]

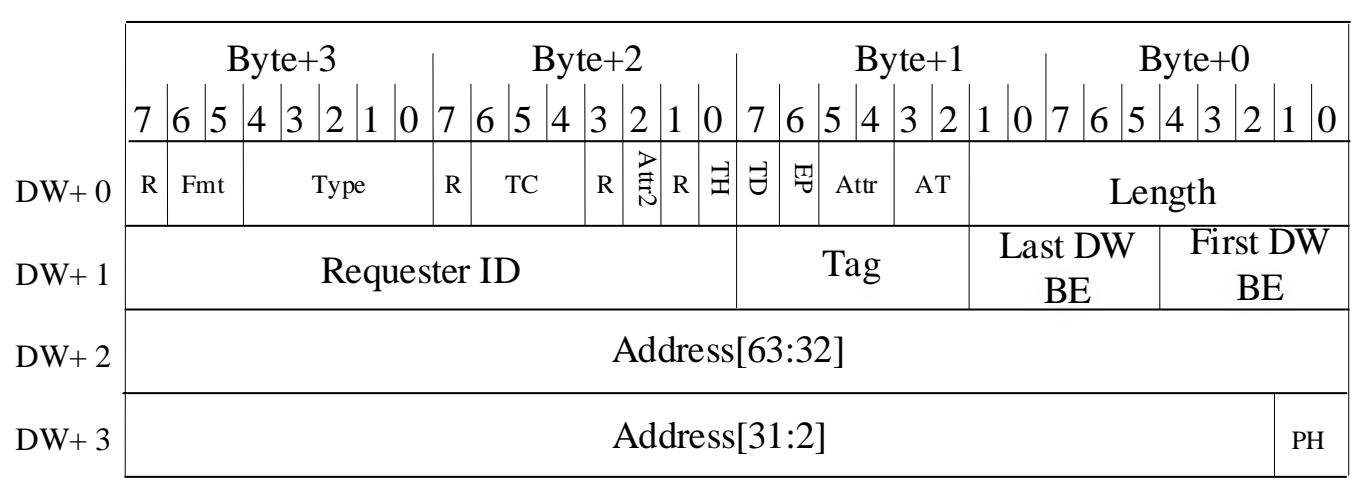

Fig 3: PCI Express Base Specification 2.0 Byte Order[2]

As the Virtex-7 PCI Express Gen3 Integrated Block consists of four interfaces based on the AXI4-Stream protocol, the completion packets from Root Complex (RC) no longer distinguished as $\mathrm{MWr}, \mathrm{Cpl}$ or CplD, all of CplD packets can be delivered through the Requester Completion (RC) Interface specially. Then the Fmt byte defined in the PCI Express specification 2.0 can be removed. Table 1 shows the Gen 3 and Gen2 transaction types.

Table 1: Gen3 Transaction Types[1]

\begin{tabular}{cccc}
\hline \multirow{2}{*}{$\begin{array}{c}\text { IP } \\
\text { cores }\end{array}$} & TLP Type & $\begin{array}{c}\text { Request Type } \\
\text { (binary) }\end{array}$ & Description \\
\cline { 3 - 3 } & & Req Type[78:75] & \\
Gen3 & MRd & 0000 & Memory Read Request \\
IP & MWr & 0001 & Memory Write Request \\
Core & IORd & 0010 & I/O Read Request \\
& IOWr & 0011 & I/O Write Request \\
\hline
\end{tabular}


Table 2: Gen2transaction Types[1]

\begin{tabular}{|c|c|c|c|c|}
\hline \multirow{2}{*}{$\begin{array}{c}\text { IP } \\
\text { cores }\end{array}$} & \multirow[t]{2}{*}{ TLP Type } & \multicolumn{2}{|c|}{$\begin{array}{l}\text { Request Type } \\
\text { (binary) }\end{array}$} & \multirow[t]{2}{*}{ Description } \\
\hline & & Fmt [2:0] & Type[4:0] & \\
\hline \multirow{6}{*}{$\begin{array}{c}\text { Gen2 } \\
\text { IP } \\
\text { Core }\end{array}$} & MRd & $\begin{array}{l}000 \\
001\end{array}$ & 00000 & Memory Read Request \\
\hline & MWr & $\begin{array}{l}010 \\
011\end{array}$ & 00000 & Memory Write Request \\
\hline & IORd & 000 & 00010 & I/O Read Request \\
\hline & IOWr & 010 & 00010 & I/O Write Request \\
\hline & Cpl & 000 & 01010 & $\begin{array}{l}\text { Completion without Data - Used for } \\
\text { I/O and Configuration Write } \\
\text { Completions with any Completion } \\
\text { Status. }\end{array}$ \\
\hline & CplD & 010 & 01010 & $\begin{array}{l}\text { Completion with Data - Used for } \\
\text { Memory, I/O, and Configuration } \\
\text { Read Completions. Also used for } \\
\text { Atomic Op Completions. }\end{array}$ \\
\hline
\end{tabular}

Data alignment mode. The TLPs transferred on the client interface consist of two parts: the descriptor and the payload data followed behind the descriptor (when it has one). When a TLP's payload is present, there are two data alignment mode to the data path:

- Dword-aligned Mode: The payload bytes are immediately follow behind the descriptor in the adjacent Dword position. For data transferred from the Completer Completion (CC) interface, the valid data is presented in lane $\mathrm{N}$ :

$$
N=(12+A \bmod 4) \bmod w[5]
$$

where Ais the starting address of the payload data being transferred in byte level, and the $\mathrm{w}$ is the interface width in byte.

- Address-aligned Mode: The payload bytes always starts in the next beat after the descriptor has transferred. For data transferred from the Completer Completion (CC) interface, the valid data is presented in laneN:

$$
N=A \bmod w[5]
$$

whereAis the memory address specified in the descriptor, $\mathrm{w}$ is the interface width in byte. If there are any gap between the descriptor and the first byte of the payload, it will be filled with null bytes.

From the above discussion, we demonstrates that theVirtex-7 PCI Express Gen3 IP core is more powerful and make the transfer much simpler.

\section{The design and implementation of PCI Express Gen3 DMA}

Our design is based on the Xilinx VC709 Connectivity Kit, and utilize the PCI Express Endpoint IP core in the xc7vx690t chip. In this design, the data could successfully transfer between the DDR3 in computer and the SO-DIMM on the VC709 development board. The details of this design as follows: 
The design of DMA controller. The Virtex-7 PCI Express Gen3 Integrated Block supplies four client interfaces to users, which could meet user's needs better for designing a transaction layer. The DMAcontroller is the key of the transaction layer design.

The DMA controller consists of 6 parts: TX Engine、RX Engine、 Tag Generator、Data Presenter、DMA Control/Status Regs and DMA DDR3 Interface, as shown in Figure 4.

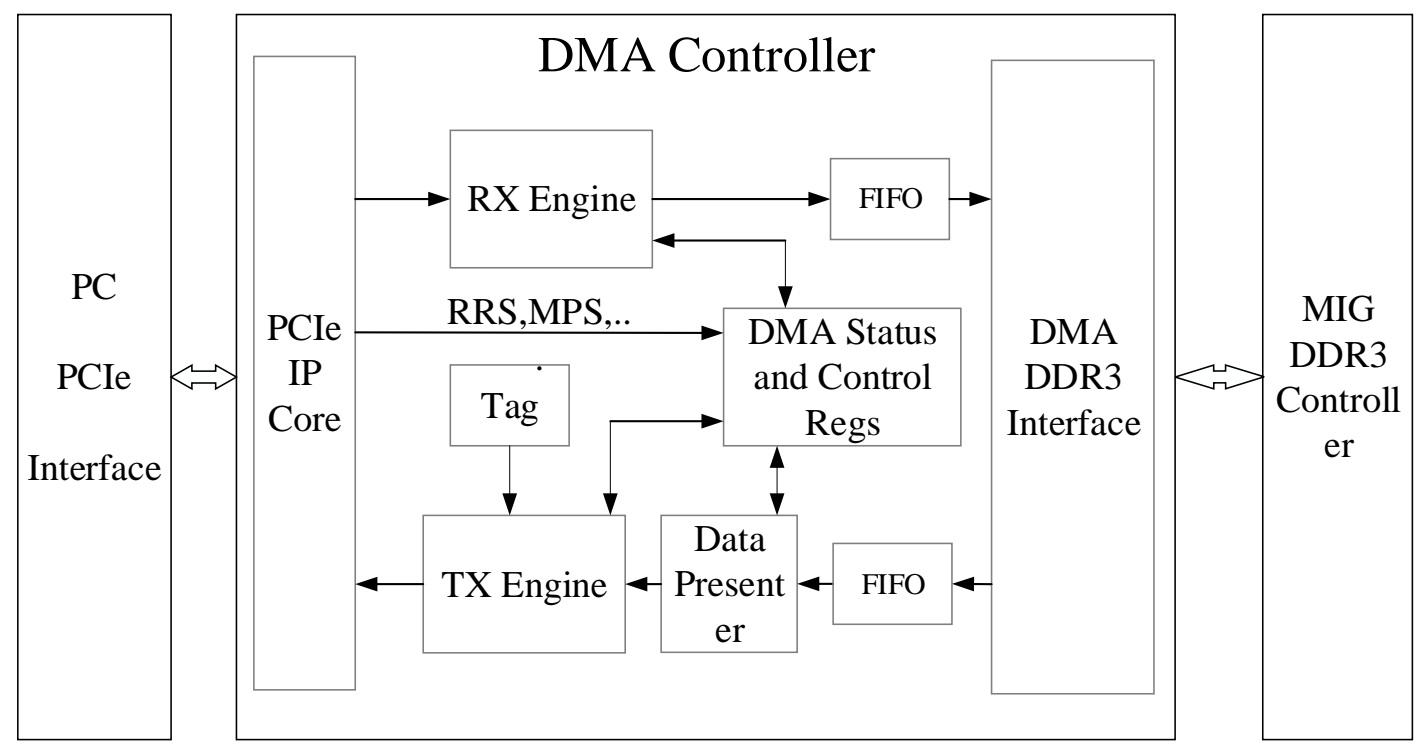

Fig 4: PCI Express Application Diagram

- RX Engine: The RX Engine receives and decodes the packets from the integrated block and routes the payload to the corresponding memory. Figure 5 shows the RX Engine State Machine.

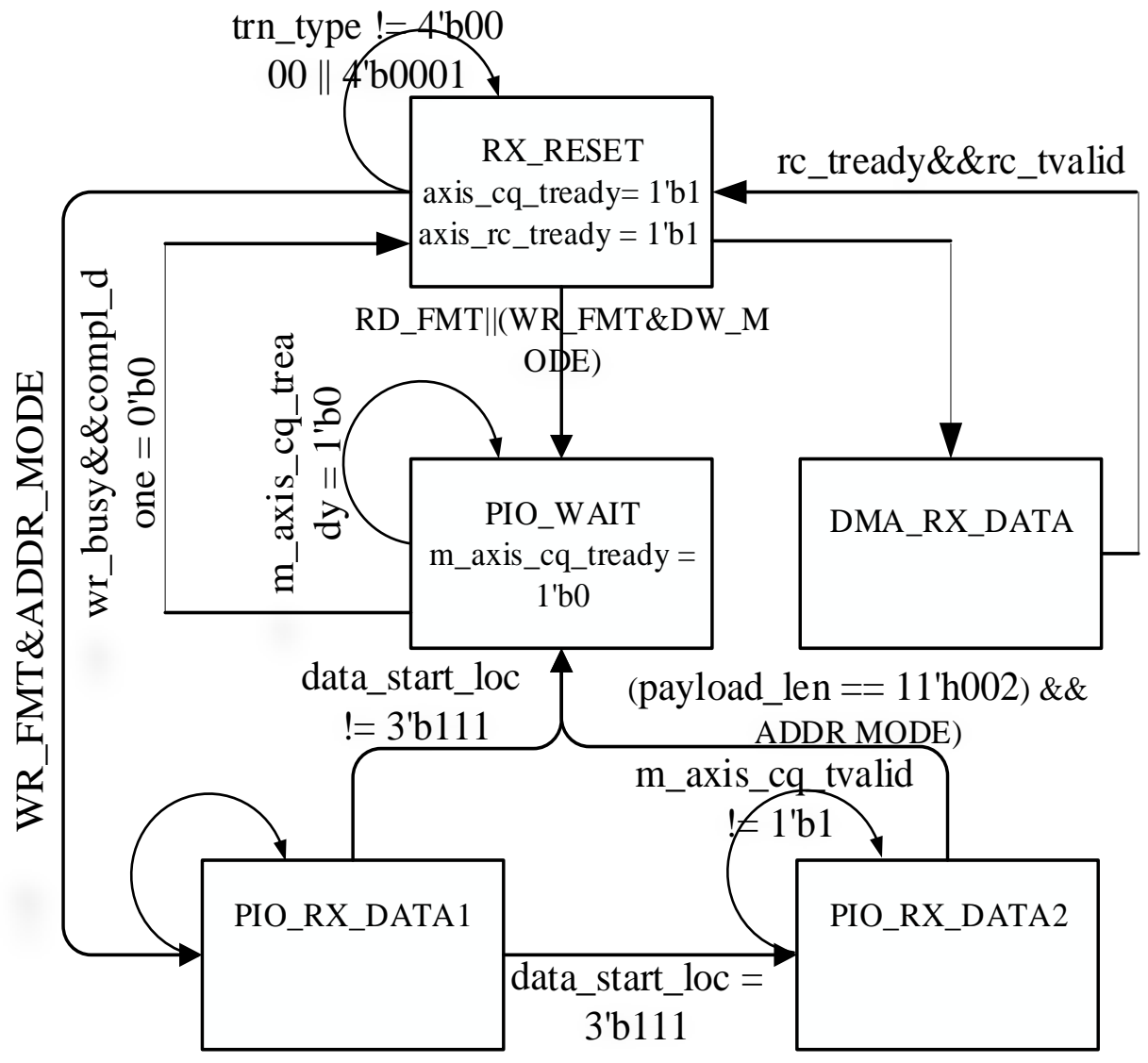

Fig 5: RX Engine State Machine 
- TX Engine: The TX Engine generates and transmits the packets through a simple stare machine to the integrated block in compliant with AXI4-Stream protocol. Figure 6 shows the RX Engine State Machine.

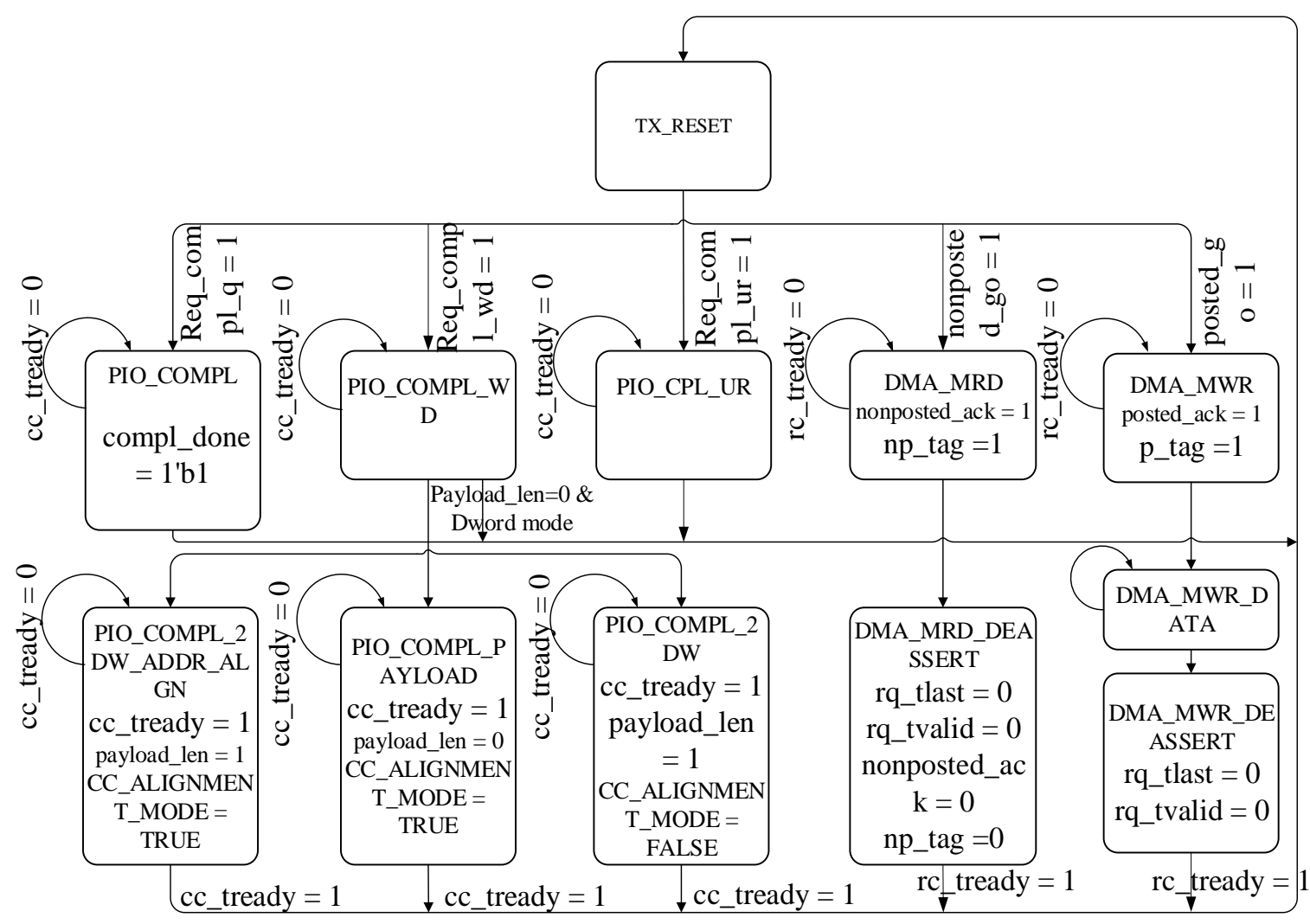

Fig 6: TX Engine State Machine

-Tag Generator: This block creates unique tags for each request packet. The tags are generated by one 8-bit counter. It is used to identify each outstanding packet in the link and the value mustn't confliction with the others[7].

-Data Presenter: This block provides 256-bit data from the DDR3 interface to the TX Engine block and utilize some handshaking signals to communicate with them.

-DMA Control/Status Regs: The DMA Control/Status Regsis mapped to the PCI memory space as the Base Address Register (BAR) 0, these regs define the DMA operations. All of these regs are 32-bit read/write access. It is the main block that the host communicates and control for DMA operations.

-DMA DDR3 Interface: The DMA DDR3 Interface provides MIG DDR3 address and other control signals, and make clock domain change between the MIG DDR3 clock domain and the DMA Controller domain.

The DMA read/write operation. The host executes a DMA read or write operation by initializing the DMA Control/Status Regs. The host sets the DMA read/write data source address, destination address, the transfer size and the start bit, then finish a complete DMA operation. The regs description are contained in Table 3. 
Table3: DMA Control/Status Register Files Definition

\begin{tabular}{|c|c|c|c|}
\hline Reg Number & Offset & Register Name & Types \\
\hline 1 & $0 \times 00$ & Read DMA operation Start & \multirow{6}{*}{$\begin{array}{c}\text { DMA Read } \\
\text { Operation } \\
\text { Regs }\end{array}$} \\
\hline 2 & $0 \times 04$ & $\begin{array}{lll}\text { Host } & \text { Memory } & \text { Source } \\
\text { Upper } & & \end{array}$ & \\
\hline 3 & $0 \times 08$ & $\begin{array}{l}\text { Host Memory } \quad \text { Source } \\
\text { Lower }\end{array}$ & \\
\hline 4 & $0 \mathrm{x} 0 \mathrm{C}$ & DDR3 Address Destination & \\
\hline 5 & $0 \times 10$ & DMA Read Transfer Size & \\
\hline 6 & $0 \times 14$ & Read DMA operation Done & \\
\hline 7 & $0 \times 18$ & Write DMA operation Start & \multirow{6}{*}{$\begin{array}{c}\text { DMA } \\
\text { Write } \\
\text { Operation } \\
\text { Regs }\end{array}$} \\
\hline 8 & $0 \times 1 C$ & $\begin{array}{l}\text { Host Memory Destination } \\
\text { Upper }\end{array}$ & \\
\hline 9 & $0 \times 20$ & $\begin{array}{l}\text { Host Memory Destination } \\
\text { Lower }\end{array}$ & \\
\hline 10 & $0 \times 24$ & DDR3 Address Source & \\
\hline 11 & $0 \times 28$ & DMA Write Transfer Size & \\
\hline 12 & $0 \times 2 C$ & $\begin{array}{l}\text { Write DMA operation } \\
\text { Done }\end{array}$ & \\
\hline
\end{tabular}

The DMA read/write operation execute as follows: (1)Initialize the DMA read/write data source register; (2) Initialize the DMA read/write data destination register; (3) Initialize the DMA read/write transfer size; (4) Set the DMA read/write start bit; (5) Reset the DMA read/write start bit and set the DMA read/write done bit. The process is shown in Figure 7.

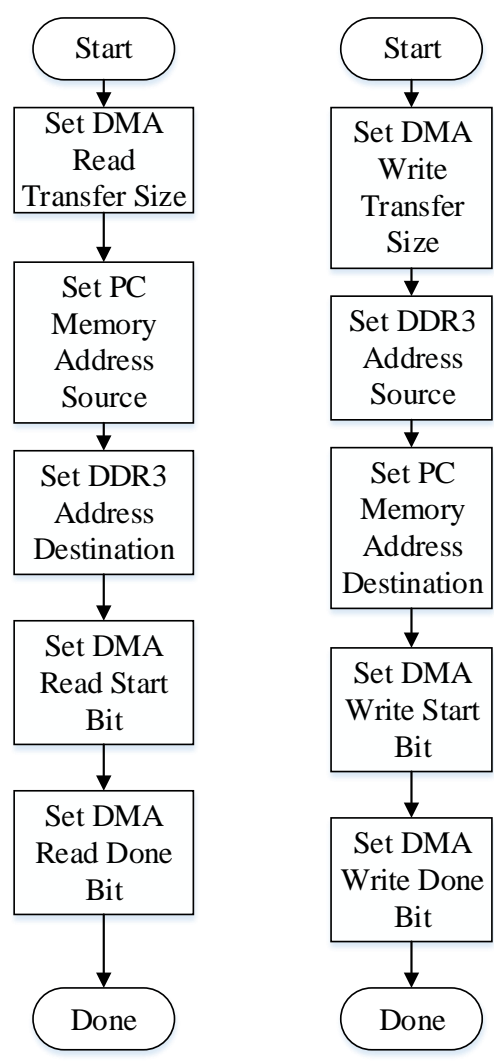

Fig 7: The DMA Read/Write Operation 


\section{Evaluation}

The VIVADO Design Suit supplies a Graphical User Interface (GUI), in which the Virtex-7 PCI Express Gen3 Integrated Block can be configured. In this design, the Integrated Block is configured as shown in Table 4.

Table 4: The Configuration of PCI Express Gen3 Integrated Block

\begin{tabular}{cc}
\hline Configuration options & Parameters \\
\hline Device/Port Type & $\begin{array}{c}\text { PCI Express Endpoint } \\
\text { device }\end{array}$ \\
\hline Lane Width & x8 \\
\hline Maximum Link Speed & $8.0 \mathrm{GT} / \mathrm{s}$ \\
\hline AXI-ST Interface Width & $256 \mathrm{bit}$ \\
\hline AXI-ST Interface Frequency & $250 \mathrm{MHz}$ \\
\hline AXI-ST Alignment Mode & Address Aligned \\
\hline Reference Clock Frequency & $250 \mathrm{MHz}$ \\
\hline PF0 Max Payload Size & $256 \mathrm{Bytes}$ \\
\hline Bar0 & $8 \mathrm{~KB}(32 \mathrm{bit})$ \\
\hline
\end{tabular}

Our design is based on the Xilinx VC709 Connectivity Kit, implemented and verified in VIVADO 2014.1 Design Suit, and tested under the Windows7 64-bit OS in DELL Vostro 270 host. The testing result is shown in Table 5 .

Table 5: The Test Result of Bandwidth

\begin{tabular}{ccc}
\hline & Test Type & Speed \\
\hline \multirow{2}{*}{ READ } & $\begin{array}{c}\text { RC Read Endpoint } \\
\text { (Single DW) }\end{array}$ & $1.5 \mathrm{MBps}$ \\
\cline { 2 - 3 } & $\begin{array}{c}\text { Endpoint Read RC } \\
\text { (DMA) }\end{array}$ & $2.89 \mathrm{GBps}$ \\
WRITE & $\begin{array}{c}\text { RC Write Endpoint } \\
\text { (Single DW) }\end{array}$ & $3.0 \mathrm{MBps}$ \\
\cline { 2 - 3 } & $\begin{array}{c}\text { Endpoint Write RC } \\
\text { (DMA) }\end{array}$ & $3.18 \mathrm{GBps}$ \\
\hline
\end{tabular}

\section{Related work}

This section summarized the prior work on the design and implementation of DMA transfer through PCI Express interface based on FPGA. Recently there has been a lot of research for designing the DMA controller through PCI Express Gen2 interface. [8], implemented a DMA reading and writing module on the Xilinx Virtex-5 based on the PCI Express 2.0 protocol. In[9], the authors have proposed a design of DMA transmission with PCI Express 2.0 interface based on the Altera Cyclonc IV GX.

Our work differs from all these prior works in that, we designed a new PCI Express Gen3 interface based on the PCI Express 3.0 protocol and designed a DMA controller based on the Xilinx Virtex-7 FPGA Gen3 Integrated Block for PCI Express. 


\section{Conclusions}

In this paper, we designed a new PCI Express Gen3 interface based on the Virtex-7 PCI Express Gen3 Integrated Block and further designed a DMA Controller based on the PCI Express Gen3 interface. The test results show that this interface could carry an average rate of $3.04 \mathrm{~GB} / \mathrm{s}$, this design provides up to $300 \%$ improvement over the similar design based on PCI Express Gen2 interface.

\section{Acknowledgements}

This work is supported in part by Open Research Fund of State Key Laboratory of High Performance Computing under Grant No.201302-01 and No.201402-01 andthe SRFDP under Grant No.20114307130003 and the National Natural Science Foundation of China under Grant No.61176030.

\section{Reference}

[1] PCI-SIG. PCI Express Base Specification Revision 3.0 [Z]. https://www.pcisig.com/ specifications/pciexpress/base $3 /$.

[2] PCI-SIG. PCI Express Base Specification Revision 2.0 [Z]. https://www.pcisig.com/ specifications/pciexpress/base2/.

[3] PCI-SIG. PCI Express 3.0 Integrators List[Z].https://www.pcisig.com/developers/compliance_ program/integrators list/pcie_3.0/.

[4] Virtex-7 FPGA XT Connectivity Targeted Reference Design for the VC709 Board. http://www.xilinx.com/support/documentation/boards_and_kits/vc709/2013_2/ug962-v7-vc709-xtconnectivity-trd-ug.pdf

[5] Virtex-7 FPGA Gen3 Integrated Block for PCI Express v3.0 LogiCORE IP Product Guide.http://www.xilinx.com/support/documentation/ip_documentation/pcie3_7x/v3_0/pg023_v7_ pcie_gen3.pdf

[6] Virtex-6 FPGA Integrated Block for PCI Express User Guide. http://www.xilinx.com/support/documentation/ip_documentation/v6_pcie/v2_5/ug671_V6_IntBloc k_PCIe.pdf

[7] MindShare, Inc, Ravi Budruk, Don Anderson, Tom Shanley. PCI Express System Architecture[M]. Addison Wesley. September 04, 2003.

[8] Jinghua Wang, Shanqing Hu. The Implementation of DMA Reading and Writing Module on FPGA Based on PCI Express Protocol[J]. Microcomputer Information,2010,26(10):7-9.

[9] Muguo Li, Ying Huang. Design of DMA Transmission with PCIe Bus Interface Based on FPGA[J]. Computer Measurement \& Control,2013.21(1):233-235. 